\title{
UNA NUEVA ESPECIE DE SISYRINCHIUM (IRIDACEAE) DEL ESTADO DE GUANAJUATO, MÉXICO
}

\author{
Jacqueline Ceja-Romero, Adolfo Espejo-Serna \\ y ANA Rosa LóPEZ-FERrari
}

Universidad Autónoma Metropolitana Iztapalapa, División de Ciencias Biológicas y de la Salud, Departamento de Biología, Herbario Metropolitano, Apdo. postal 55-535, 09340 México, D.F., México.jcr@xanum.uam.mx; aes@xanum.uam.mx

\section{RESUMEN}

Se describe e ilustra a Sisyrinchium guanajuatense, conocida hasta ahora del estado de Guanajuato, México. El nuevo taxon pertenece al subgénero Echthronema y se compara con algunas especies del complejo de S. schaffneri, con las cuales está relacionado. Se incluye una clave para identificar los taxones mencionados.

Palabras clave: Guanajuato, Iridaceae, México, Sisyrinchium.

\begin{abstract}
Sisyrinchium guanajuatense is described and illustrated. The new species is known only from the state of Guanajuato, Mexico. It belongs to the subgenus Echthronema and is compared with some species of the $S$. schaffneri complex to which it relates. An identification key for all these taxa is included.
\end{abstract}

Key words: Guanajuato, Iridaceae, Mexico, Sisyrinchium.

Sisyrinchium L. (Iridaceae) es un género del nuevo mundo que incluye entre 60 y 200 especies agrupadas en dos subgéneros, Echthronema y Sisyrinchium (Rudall et al., 1986; Henrich y Goldblatt, 1987; Calderón de Rzedowski, 1988; McVaugh, 1989; Calderón de Rzedowski y Rzedowski, 1990; Goldblatt et al., 1990; Espejo y López Ferrari, 1990, 1994). Dentro de Echthronema se agrupan las especies de flores amarillas con la corola extendida y los filamentos parcialmente connados. En 
el subgénero Sisyrinchium se incluyen los taxones de flores azules a blancas con el centro amarillo, la corola cupuliforme y los filamentos generalmente unidos casi en su totalidad formando una larga columna estaminal. De México se tienen registrados 44 taxones, 30 del primer subgénero y 14 del segundo, de los cuales 17(38\%) son endémicos (Espejo y López-Ferrari, 1996; Ceja-Romero et al., 1998; Espejo et al., 1998, 1999, 2001).

La taxonomía del género es sumamente confusa, ya que las especies que lo integran son muy variables y los caracteres útiles para el reconocimiento de las mismas generalmente se pierden durante el proceso de herborización. Esto ha contribuido a la gran disparidad en el número de taxones reconocidos.

Durante la revisión del complejo de especies de Sisyrinchium schaffneri S. Watson (subgénero Echthronema) (Ceja-Romero, 2007), encontramos un grupo de ejemplares cuyo análisis nos hizo sospechar que podría representar una entidad no descrita. Con el fin de aclarar esta situación, recolectamos ejemplares del taxon en cuestión, elaboramos disecciones florales, fotografiamos las plantas en su hábitat y medimos diversos caracteres morfológicos. Tal información se complementó con datos sobre la anatomía de la lámina foliar. Los datos generados fueron comparados con Sisyrinchium cernuum (E. P. Bicknell) Kearney, S. schaffneri, S. tinctorium Kunth y $S$. translucens (E. P. Bicknell) Espejo \& López-Ferrari. Después de su análisis, llegamos a la conclusión de que los ejemplares en cuestión representan a una nueva especie que aquí proponemos:

Sisyrinchium guanajuatense Ceja, Espejo et López-Ferrari, sp. nov. Fig. 1.

Herbae perennes, erectae, 19-26 cm altae; folia omnia basalia, disticha, aequitantia, lineari-ensiformia, glabra, purpurea, basim versus $15-22 \mathrm{~cm}$ longa, folia vetera annulum ex fibris constatum basim versus formantia; scapus simplex, alatus, glaber, 17-24 cm longus, 0.5-1 mm latus, margine denticulatus versus apicem; spathae inaequales, geniculatae; tepala flava, elliptica, $8-10 \mathrm{~mm}$ longa, $2.5-4.5 \mathrm{~mm}$ lata; capsula nutans, sphaerica vel subsphaerica, glabra, 3.5-5 mm longa, 3-4 mm diametro; semina sphaerica vel subsphaerica, brunnea, 1-1.5 mm diametro, reticulata.

Hierba perenne, erecta, de 19 a $26 \mathrm{~cm}$ de alto; raíces fibrosas de 0.5 a $1 \mathrm{~mm}$ de diámetro; hojas todas basales, dísticas, equitantes, linear-ensiformes, rectas, glabras, con las bases purpúreas, de 15 a $22 \mathrm{~cm}$ de largo y 1 a $2.5 \mathrm{~mm}$ de ancho, el margen denticulado, las hojas viejas formando un collar de fibras en la base del escapo; escapo simple, alado, glabro, de 17 a $24 \mathrm{~cm}$ de largo y 0.5 a $1 \mathrm{~mm}$ de an- 

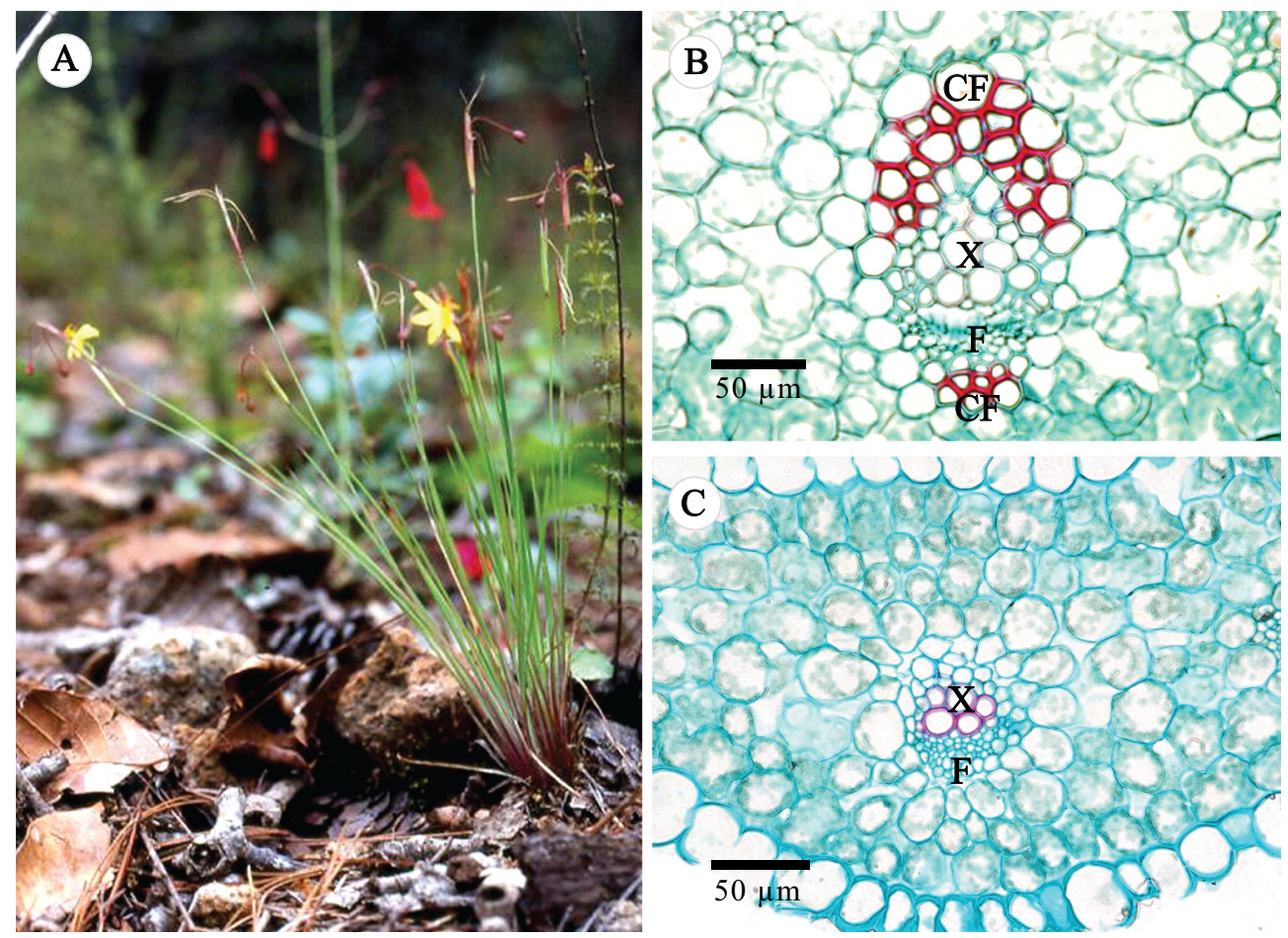

Fig. 1. A. hábito de Sisyrinchium guanajuatense Ceja, Espejo et López-Ferrari; B. haz vascular de S. guanajuatense; C. haz vascular de S. schaffneri S. Watson. (X: xilema; F: floema; CF: casquete de fibras).

cho, el margen denticulado hacia el ápice; ripidio terminal cubierto por dos brácteas espatáceas desiguales, conduplicadas, generalmente geniculadas, fusionadas en la base, la externa más larga que la interna, de 2.1 a $3 \mathrm{~cm}$ de largo y 1.5 a $2.5 \mathrm{~mm}$ de ancho, el margen entero a denticulado, con tintes purpúreos, la interna de 2 a $2.3 \mathrm{~cm}$ de largo y 1 a $2 \mathrm{~mm}$ de ancho, el margen hialino-purpúreo; brácteas florales hialinas; pedicelos filiformes, erectos en la floración, reflexos en la fructificación, de 11 a 16 $\mathrm{mm}$ de largo; una flor abierta por ripidio, exserta, el perianto extendido, los tépalos amarillos con venas pardas, iguales a subiguales, elípticos, de 8 a $10 \mathrm{~mm}$ de largo y 2.5 a $4.5 \mathrm{~mm}$ de ancho; filamentos unidos en la base formando un tubo de 1 a 1.5 $\mathrm{mm}$ de alto, la parte libre de 2.5 a $3.5 \mathrm{~mm}$ de largo; anteras amarillas, de 3 a $3.5 \mathrm{~mm}$ largo; estilo trífido, las ramas alternas con las anteras, amarillas, de 3 a $3.5 \mathrm{~mm}$ de largo, estigma capitado, amarillo, polen amarillo; ovario glabro, turbinado, de 1.5 a 
$2.5 \mathrm{~mm}$ de largo y 1 a $1.5 \mathrm{~mm}$ de ancho; cápsulas péndulas, generalmente esféricas a subesféricas, glabras, de 3.5 a $5 \mathrm{~mm}$ de largo por 3 a $4 \mathrm{~mm}$ de ancho; semillas globosas a subglobosas, pardas, umbilicadas, de alrededor de 1 a $1.5 \mathrm{~mm}$ de diámetro, con la testa reticulada.

Tipo: México, Guanajuato, municipio San Luis de la Paz, 1 km después de Charco Azul rumbo a San Agustín, sobre la carretera Xichú - San Luis de la Paz, J. Ceja et al. 479 (Holótipo: UAMIZ; isótipos: CIIDIR, IEB).

Descripción anatómica de la lámina foliar. Células epidérmicas intercostales de 402 a $408 \mu \mathrm{m}$ de largo y 19 a $24 \mu \mathrm{m}$ de ancho, las costales de 811 a 818 $\mu \mathrm{m}$ de largo y 18 a $19 \mu \mathrm{m}$ de ancho. Estomas anomocíticos, células oclusivas por debajo del nivel de las epidérmicas, de 41 a $43 \mu \mathrm{m}$ de largo; contorno de la lámina en corte transversal lateral aplanado; epidermis uniestratificada, con las células de 18 a $28 \mu \mathrm{m}$ de alto y 15 a $19 \mu \mathrm{m}$ de ancho, las intercostales con la pared de ca. 3 $\mu \mathrm{m}$ de grueso, las costales de ca. $6 \mu \mathrm{m}$ de grueso y las marginales de 7 a $9 \mu \mathrm{m}$ de grueso; parénquima clorofílico con células isodiamétricas; parénquima esponjoso con células isodiamétricas a ligeramente lobuladas; haces vasculares 12 a 15, de tres tamaños (órdenes), los de primero de alrededor de 82 a $103 \mu \mathrm{m}$ de largo, los de segundo de 49 a $72 \mu \mathrm{m}$ largo, los de tercero de 19 a $30 \mu \mathrm{m}$; vaina parenquimática rodeando a los haces vasculares, casquete de fibras en los polos del xilema y del floema.

Ejemplares examinados: Guanajuato: municipio de Guanajuato, $5.2 \mathrm{~km}$ después de Santa Rosa, rumbo a Guanajuato, J. Ceja et al. 491 (UAMIZ); $6.2 \mathrm{~km}$ después de Guanajuato rumbo a Dolores Hidalgo, en la cuenca de La Esperanza, J. Ceja 266 (UAMIZ); 8 km después de Santa Rosa, rumbo a Dolores Hidalgo, J. Ceja et al. R. 483 (UAMIZ); municipio de San Luis de la Paz, 10 km al NW de Mesas de Jesús, J. Rzedowski 52848 (UAMIZ); municipio de Victoria, 1 km después de Charco Azul rumbo a San Agustín, sobre la carretera Xichú - San Luis de la Paz, J. Ceja et al. 479 (UAMIZ); El Charco Azul, Sierra de Xichú, E. Ventura y E. López 9609 (IEB, UAMIZ); Charco Azul, E. Ventura y E. López 8652 (IEB, UAMIZ); alrededores de San Agustín, J. Rzedowski 50824 (IEB, UAMIZ); $25 \mathrm{~km}$ al W de Xichú, sobre la carretera a San Luis de la Paz, J. Rzedowski 44836 (IEB).

Sisyrinchium guanajuatense se conoce sólo de la porción central y nororiental del estado de Guanajuato, donde crece en lugares abiertos dentro de bosques de pino 
y de encino. Florece de julio y agosto. El intervalo altitudinal en el que crece va de 2200 a $2430 \mathrm{~m}$.

Los ejemplares previamente recolectados del nuevo taxon habían sido identificados como Sisyrinchium schaffneri. Sin embargo, existen diferencias morfológicas y anatómicas entre ambas especies. Los individuos de $S$. guanajuatense se caracterizan por el collar basal de fibras formado por los restos de las hojas de la temporada anterior, por el color purpúreo en la base de las hojas, por las espatas generalmente geniculadas y por sus frutos esféricos a subesféricos (Fig. 1A). Anatómicamente, las láminas foliares de $S$. guanajuatense presentan ocasionalmente papilas en la epidermis, en corte transversal y los haces vasculares tienen casquetes de fibras tanto en el polo del xilema como en el del floema (Fig. 1B). Los individuos de $S$. schaffneri no forman un collar de fibras en la base de la planta, el escapo es ligeramente más ancho, las espatas generalmente no se geniculan y los frutos son elipsoides a turbinados. Las láminas foliares de S. schaffneri, son lisas en vista superficial y carecen de esclerénquima en los polos de los haces vasculares (Fig. 1C). Dentro del complejo de Sisyrinchium schaffneri, existen otros taxones con los cuales la especie nueva está relacionada. En el Cuadro 1 pueden observarse las características de cada uno de ellos y a continuación se presenta una clave para su identificación.

1. Tépalos de hasta $0.5 \mathrm{~cm}$ de largo, anteras de menos de $2 \mathrm{~mm}$ de largo

S. cernuum

1. Tépalos de más de $0.5 \mathrm{~cm}$ de largo; anteras de más de $2 \mathrm{~mm}$ de largo.

2. Espata externa de hasta $3 \mathrm{~cm}$ de largo; proporción espata externa/espata interna menor de 1.5 .

3. Escapo floral de 1.5 a $3 \mathrm{~mm}$ de ancho; lámina con los haces vasculares dispuestos en dos hileras

S. tinctorium

3. Escapo floral de 0.5 a $1.5 \mathrm{~mm}$ de ancho; lámina con los haces vasculares dispuestos en una hilera.

4. Plantas hasta de $20 \mathrm{~cm}$ de alto; frutos elipsoides a turbinados; haces vasculares sin esclerénquima en los polos del xilema y del floema

S. schaffneri

4. Plantas de 19 a $26 \mathrm{~cm}$ de alto; frutos esféricos a subesféricos; haces vasculares con esclerénquima en los polos del xilema y del floema ......

S. guanajuatense

2. Espata externa de al menos $4 \mathrm{~cm}$ de largo; proporción espata externa/espata interna mayor de 2

S. translucens 


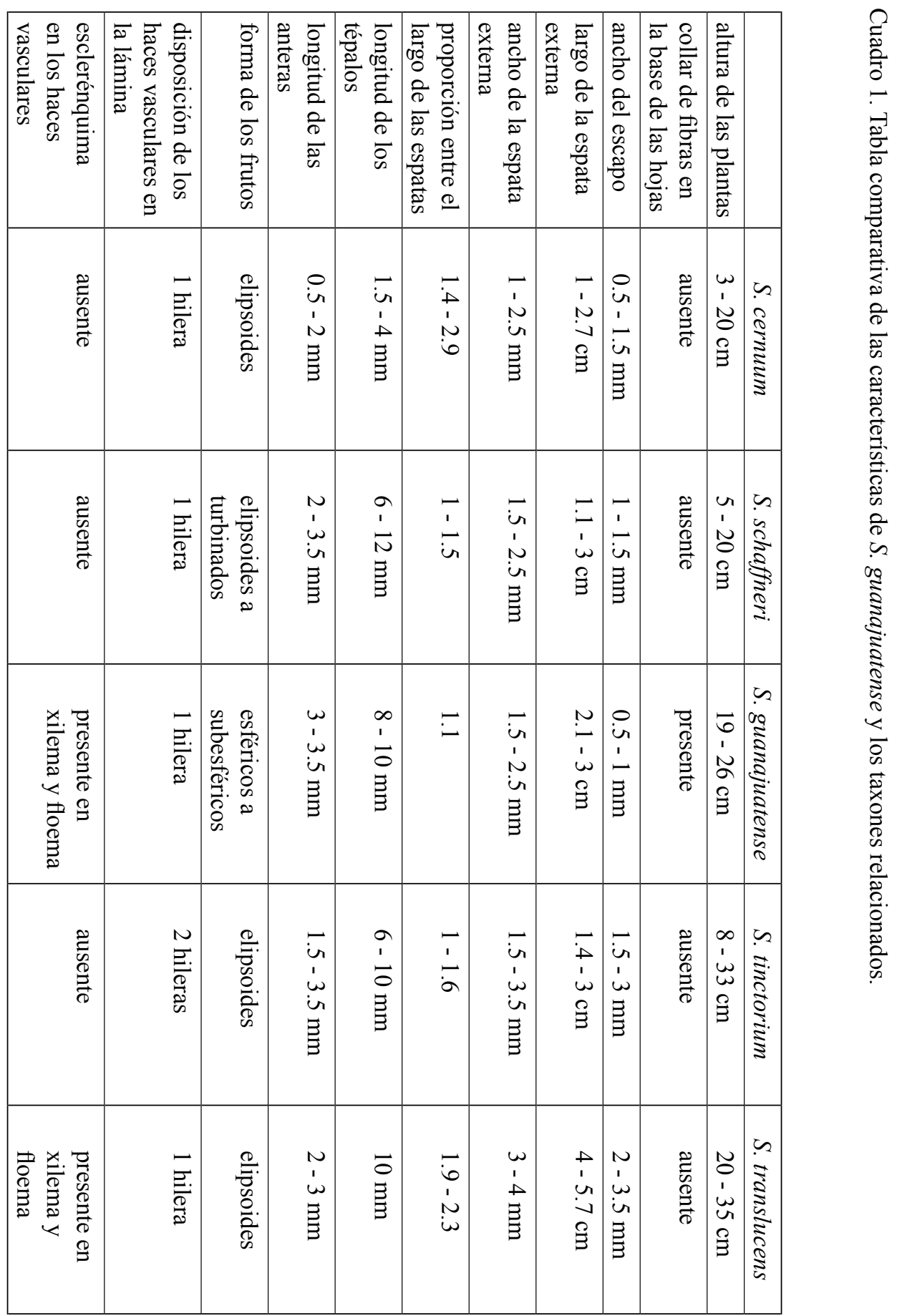




\section{AGRADECIMIENTOS}

Agradecemos a Jerzy Rzedowski y a Graciela Calderón la revisión crítica del manuscrito. Nuestro agradecimiento para Aniceto Mendoza Ruiz por su apoyo invaluable e incondicional en el trabajo de campo. Estamos agradecidos con los curadores de los herbarios BCMEX, CAS, CHAPA, CIIDIR, ENCB, GH, HCIB, IBUG, IEB, MEXU, MICH, MO, NY, POM, RSA, SLPM, TEX, UAMIZ, UC, US, $\mathrm{XAL}$, por las facilidades otorgadas para la consulta de material. Los resultados de este trabajo son parte de la tesis de Maestría en Ciencias (Biología Vegetal) de la primera autora.

\section{LITERATURA CITADA}

Calderón de Rzedowski, G. 1988. Algunas consideraciones acerca de la familia Iridaceae en el Valle de México. Acta Bot. Mex. 1: 21-27.

Calderón de Rzedowski, G. y J. Rzedowski. 1990. Iridaceae. In: Rzedowski, J. y G. Calderón de Rzedowski (eds.). Flora fanerogámica del Valle de México. Vol III. Instituto de Ecología, A.C. Pátzcuaro, Michoacán. pp 323-340.

Ceja-Romero, J. 2007. Revisión taxonómica de las especies mexicanas del complejo de Sisyrinchium schaffneri $\mathrm{S}$. Watson (Iridaceae). Tesis de Maestría en Ciencias (Biología Vegetal). Facultad de Ciencias, Universidad Nacional Autónoma de México. México, D.F. 118 pp.

Ceja-Romero, J., A. Espejo y A. R. López-Ferrari. 1998. Sisyrinchium arguellesiae (Iridaceae: Sisyrinchieae) una nueva especie del estado de Querétaro. Acta Bot. Mex. 44: 87-91.

Espejo, A. y A. R. López-Ferrari. 1990. Las monocotiledóneas mexicanas. Una sinopsis florística. Parte VI. Consejo Nacional de la Flora de México, A.C. Universidad Autónoma Metropolitana-Iztapalapa, Comisión Nacional para el Conocimiento y Uso de la Biodiversidad. México, D.F. 116 pp.

Espejo, A. y A. R. López-Ferrari. 1994. Iridaceae. Flora de Veracruz 82: 1-58.

Espejo, A. y A. R. López-Ferrari. 1996. Comentarios florístico-ecológicos sobre las iridáceas mexicanas. Acta Bot. Mex. 34: 25-47.

Espejo, A., A. R. López-Ferrari y J. Ceja-Romero. 1998. Una nueva especie gipsófila de Sisyrinchium (Iridaceae: Sisyrinchieae) de México. Acta Bot. Mex. 45: 43-47.

Espejo, A., A. R. López-Ferrari y J. Ceja-Romero. 1999. Una nueva especie de Sisyrinchium (Iridaceae: Sisyrinchieae) del estado de Durango, México. Acta Bot. Mex. 49: 20-22.

Espejo, A., A. R. López-Ferrari, J. Ceja-Romero y A. Cholewa. 2001. Two new species of Mexican Iridaceae. Acta Bot. Mex. 55: 21-27.

Goldblatt, P., P. Rudall y E. Henrich. 1990. The genera of the Sisyrinchium alliance (Iridaceae: Iridoideae): phylogeny and relationships. Syst. Bot. 15: 497-510. 
Henrich, J. E. y P. Goldblatt. 1987. Mesoamerican Sisyrinchium (Iridaceae): new species and records, and notes on typification. Ann. Missouri Bot. Gard. 74: 903-910.

McVaugh, R. 1989. Iridaceae. Flora Novo-Galiciana 15: 294-347.

Rudall, P., A. Y. Kenton y T. J. Lawrence 1986. An anatomical and chromosomal investigation of Sisyrinchium and allied genera. Bot. Gaz. 147: 466-477.

Recibido en noviembre de 2008.

Aceptado en febrero 2009. 